\title{
Conjunctive Query Answering in Finitely-valued Fuzzy Description Logics ${ }^{\star}$
}

\author{
Theofilos Mailis ${ }^{1}$, Rafael Peñaloza ${ }^{1,2}$, and Anni-Yasmin Turhan ${ }^{1}$ \\ 1 Chair for Automata Theory, Theoretical Computer Science, TU Dresden, Germany \\ 2 Center for Advancing Electronics Dresden \\ mailis,penaloza,turhan@tcs.inf.tu-dresden.de
}

\begin{abstract}
Fuzzy Description Logics (DLs) generalize crisp ones by providing membership degree semantics for concepts and roles. A popular technique for reasoning in fuzzy DL ontologies is by providing a reduction to crisp DLs and then employ reasoning in the crisp DL. In this paper we adopt this approach to solve conjunctive query (CQ) answering problems for fuzzy DLs. We give reductions for Gödel, and Łukasiewicz variants of fuzzy $\mathcal{S R O} \mathcal{I} \mathcal{Q}$ and two kinds of fuzzy CQs. The correctness of the proposed reduction is proved and its complexity is studied for different

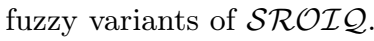

\section{Introduction}

Description Logics (DLs) are a class of knowledge representation languages with well-defined semantics that are widely used to represent the conceptual knowledge of an application domain in a structured and formally well-understood way. DLs have been successfully employed to formulate ontologies for several knowledge domains such as bio-medical applications. DLs provide the formal foundation for the standard web ontology language OWL, a milestone for the Semantic Web. In this paper we focus on the DL $\mathcal{S R O I} \mathcal{Q}$, the DL underlying (full) OWL 2.

DLs represent knowledge by means of concepts that correspond to sets of objects, and roles that relate pairs of objects. Ontology axioms are used to restrict the possible interpretations of our domain of interest. For example, we can express the fact that a CPU cpuA is overutilized and that a server that has a part that is overutilized is a server with limited resources by stating:

$$
\begin{gathered}
(\text { CPU } \sqcap \text { Overutilized })(\text { cpuA }) \\
\text { Server } \sqcap \exists \text { hasPart.Overutilized } \sqsubseteq \text { ServerWithLimitedResources }
\end{gathered}
$$

Some applications require to describe sets for which there exists no sharp, unambiguous distinction between the members and nonmembers. In our running example Overutilized is such a notion. We can say that cpuA is overutilized to

\footnotetext{
* Partially supported by DFG SFB 912 (HAEC) and the Cluster of Excellence 'cfAED'.
} 
Table 1. Families of fuzzy logic operators.

\begin{tabular}{cllll}
\hline Family & t-norm $a \otimes b$ & t-conorm $a \oplus b$ & negation $\ominus a$ & implication $\alpha \Rightarrow b$ \\
\hline Gödel & $\min (a, b)$ & $\max (a, b)$ & $\begin{cases}1, & a=0 \\
0, & a>0\end{cases}$ & $\begin{cases}1, & a \leqslant b \\
b, & a>b\end{cases}$ \\
Eukasiewicz & $\max (a+b-1,0)$ & $\min (a+b, 1)$ & $1-a$ & $\min (1-a+b, 1)$ \\
\hline
\end{tabular}

a certain degree Overutilized (cpuA $) \geqslant 0.8$. To represent this kind of information faithfully, fuzzy variants of DLs were introduced. Fuzzy DLs generalize crisp DLs by providing membership degree semantics for their concepts and roles by fuzzy sets. The membership degree of an individual to a fuzzy concept can be understood as a weight extending the logic with the possibility of expressing imprecision. Likewise, axioms describing the domain knowledge are equipped with a weight that gives additional flexibility in the restrictions of the membership degrees used. In fuzzy DLs, all crisp set operations are extended to the fuzzy case. The intersection, union, complement and implication set operations are performed by a t-norm function $\otimes$, a t-conorm function $\oplus$, a negation function $\ominus$, and an implication function $\rightarrow$, respectively. These functions or fuzzy operators are grouped in families, also simply called fuzzy logics. It is well known that different families of fuzzy operators lead to fuzzy DLs with different properties. In this paper we concentrate on the families of fuzzy logic operators displayed in Table 1 . We use the prefixes $f_{\mathrm{G}}$ and $f_{\mathrm{Ł}_{n}}$ to distinguish between Gödel and Łukasiewicz based semantics. We investigate the reasoning task of conjunctive query answering in these settings. Conjunctive queries are a very powerful way to access the facts in the ontology and it has been widely studied in the recent years for crisp DLs. We are considering finitely-valued fuzzy DLs, since unrestricted fuzzy DLs easily turn undecidable $[1,8]$. An alternative to implementing a fuzzy DL reasoner from scratch is to reduce reasoning within fuzzy DLs to reasoning in crisp DLs, which allows for the use of existing DL reasoners and to benefit from new optimizations implemented in these systems.

Although there has been a significant amount of work regarding the reduction from fuzzy to crisp DLs, this body of work concentrates mainly on the following problems: instance checking or concept satisfiability $[4,23]$. In this paper we extend these reductions to the interesting problem of conjunctive query answering. By which we can answer queries that ask for all pairs of servers and CPUs such that the CPU is a part of the server and also is over utilized to an at least 0.6 degree:

$$
\text { Server }(x) \geqslant 1 \wedge \operatorname{hasPart}(x, y) \geqslant 1 \wedge \mathrm{CPU}(y) \geqslant 1 \wedge \operatorname{Overutilized}(y) \geqslant 0.6
$$

The contributions made in this paper are the following:

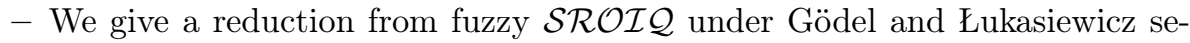
mantics to $\mathcal{S R O I} \mathcal{Q}$ for answering conjunctive queries in the finitely-valued 
setting and prove its correctness. The presented proof builds on the reductions presented in $[2,3,4]$.

- We prove that, if there exists a reduction from the fuzzy DL $f$ - $\mathcal{L}$ to the corresponding crisp DL $\mathcal{L}$ and there exists an algorithm for conjunctive query answering w.r.t. $\mathcal{L}$, then it can also be applied to answer conjunctive queries w.r.t. $f-\mathcal{L}$ in the finitely-valued setting.

- We assess the complexity of the presented conjunctive query answering technique for different fuzzy extensions of the DL $\mathcal{S H \mathcal { L }}$. $\mathcal{S H \mathcal { I }}$ is a sublan-

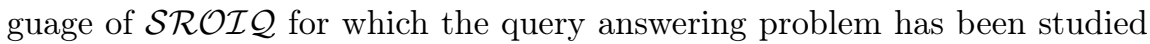
and solved [12].

- Finally, in order to ensure the correctness of our approach, we have extended the correctness proof sketched in [4] for the Lukasiewicz based extension of $\mathcal{S R O I Q}$ (for a detailed proof see the technical report accompanying this paper [16]).

The rest of the paper is structured as follows: Section 2 presents the syntax and

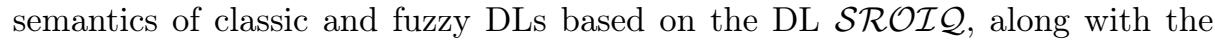
reduction procedure from the fuzzy to the crisp DL. Section 3 defines the different types of conjunctive queries in the fuzzy setting, while Section 4 presents the actual reduction from fuzzy to crisp conjunctive query answering, along with a proof of its correctness. Finally, Section 5 presents the current literature on reduction techniques and conjunctive query answering for fuzzy DLs, while Section 6 gives an overview of the paper and refers to future work and implementations.

\section{Preliminaries}

We start with a brief introduction to DL syntax and semantics and present the DL $\mathcal{S} \mathcal{R O} \mathcal{I} \mathcal{Q}$ [13]. This specific DL was chosen since: it is one of the most expressive decidable DLs, it provides the direct model-theoretic semantics of OWL 2, and there exists a reduction technique from fuzzy to classic $\mathcal{S R O I Q}$ ontologies $[2,4,23]$. DL ontologies are constructed from countable, and pairwise disjoint sets of individual names $N_{I}$, of concept names $N_{C}$, and of role names $N_{R}$. Individuals correspond to elements of the domain, concept names are used to describe sets of elements, and role names describe binary relations between elements. The set $N_{S}$ is the subset of $N_{R}$ containing only simple roles. Based on these, concept and role descriptions can be built using different constructors.

In the first and the second column of Table 2 we see most of the concept and role constructors of the highly expressive DL $\mathcal{S R O I} \mathcal{I}$-for a more detailed presentation of the crisp $\mathcal{S R O I} \mathcal{Q}$ language the reader may refer to [13], while the Gödel and Eukasiewicz fuzzy variants of the language are thoroughly presented in $[4,23]-$. In Tables $2-4$ we have the following notation: $o_{1}, \ldots, o_{m}, a, b \in N_{I}$; $r, r_{1}, \ldots, r_{n} \in N_{R} ; s \in N_{S} ; d_{1}, \ldots, d_{m}, d \in(0,1]_{\mathcal{O}}, C, D$ correspond to concept descriptions, while $\triangleright \in\{\geqslant,>\}$ and $\bowtie \in\{\leqslant,<, \geqslant,>\}$. As usual the simplest form of a concept description is an element $A \in N_{C}$. 
Table 2. Concept constructors from $\mathcal{S R O I Q ~}$

\begin{tabular}{|c|c|c|c|}
\hline & Syntax & Crisp Semantics & Fuzzy Semantics \\
\hline $\begin{array}{l}\text { Conjunction } \\
\text { Disjunction } \\
\text { Negation } \\
\text { Value restriction }\end{array}$ & $\begin{array}{l}C \sqcap D \\
C \sqcup D \\
\neg C \\
\forall r . C\end{array}$ & $\begin{array}{l}C^{\mathcal{I}} \cap D^{\mathcal{I}} \\
C^{\mathcal{I}} \cup D^{\mathcal{I}} \\
\Delta^{\mathcal{I}} \backslash C^{\mathcal{I}} \\
\left\{x \mid \forall y,(x, y) \notin R^{\mathcal{I}} \text { or } y \in C^{\mathcal{I}}\right\}\end{array}$ & $\begin{array}{l}C^{\mathcal{I}}(x) \otimes D^{\mathcal{I}}(x) \\
C^{\mathcal{I}}(x) \oplus D^{\mathcal{I}}(x) \\
\ominus C^{\mathcal{I}}(x) \\
\inf \left\{R^{\mathcal{I}}(x, y) \Rightarrow C^{\mathcal{I}}(y)\right\}\end{array}$ \\
\hline Existential restr. & $\exists r . C$ & $\left\{x \mid \exists y,(x, y) \in R^{\mathcal{I}}\right.$ and $\left.y \in C^{\mathcal{I}}\right\}$ & $\sup _{y \in \Delta^{\mathcal{I}}}\left\{R^{\mathcal{I}}(x, y) \otimes C^{\mathcal{I}}(y)\right\}$ \\
\hline $\begin{array}{l}\text { Nominals } \\
\text { fuzzy }\end{array}$ & $\begin{array}{l}\{o\} \\
\{d / o\}\end{array}$ & $\left\{o^{\mathcal{I}}\right\}$ & $\begin{array}{l}1 \text { if } x \in\{o\}, 0 \text { otherwise } \\
\sup \left\{d \mid x=o^{\mathcal{I}}\right\}\end{array}$ \\
\hline At-least restr. & $\geq n s . C$ & $\begin{array}{l}\left\{x \mid \sharp\left\{y:(x, y) \in s^{\mathcal{I}} \text { and }\right.\right. \\
\left.\left.\qquad y \in C^{\mathcal{I}}\right\} \geqslant n\right\}\end{array}$ & $\begin{aligned} \sup _{y_{1}, \ldots y_{n} \in \Delta^{\mathcal{I}}} & \left(\min _{i=1}^{n}\left\{s^{\mathcal{I}}\left(x, y_{i}\right) \otimes C^{\mathcal{I}}\left(y_{i}\right)\right\}\right) \\
\otimes & \left(\otimes \quad\left\{y_{i} \neq y_{k}\right\}\right)\end{aligned}$ \\
\hline At-most restr. & $\leq n s . C$ & $\begin{array}{l}\left\{x \mid \sharp\left\{y:(x, y) \in s^{\mathcal{I}} \text { and }\right.\right. \\
\left.\left.\qquad y \in C^{\mathcal{I}}\right\} \leqslant n\right\}\end{array}$ & 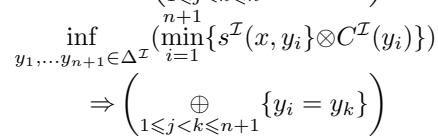 \\
\hline
\end{tabular}

Table 3. $\mathcal{S R O} \mathcal{I} \mathcal{Q}$ TBox axioms.

\begin{tabular}{llll}
\hline & Syntax & Crisp Semantics & Fuzzy Semantics \\
\hline GCI & $C \sqsubseteq D$ & $C^{\mathcal{I}} \subseteq D^{\mathcal{I}}$ & $\inf _{x \in \Delta^{\mathcal{I}}}\left\{C^{\mathcal{I}}(x) \Rightarrow D^{\mathcal{I}}(x)\right\}=1$ \\
$\quad$ fuzzy & $\langle C \sqsubseteq D, \triangleright d\rangle$ & - & $\inf _{x \in \Delta^{\mathcal{I}}}\left\{C^{\mathcal{I}}(x) \Rightarrow D^{\mathcal{I}}(x)\right\} \triangleright d$ \\
RI & $r_{1} \ldots r_{n} \sqsubseteq r$ & $r_{1}^{\mathcal{I}} \circ \ldots \circ r_{n}^{\mathcal{I}} \subseteq r^{\mathcal{I}}$ & $\inf _{x, y \in \Delta^{\mathcal{I}}}\left\{\left[r_{1}^{\mathcal{I}} \circ^{\otimes} \ldots \circ^{\otimes} r_{n}^{\mathcal{I}}\right](x, y) \Rightarrow r^{\mathcal{I}}(x, y)\right\}=1$ \\
$\quad$ fuzzy & $\left\langle r_{1} \ldots r_{n} \sqsubseteq r \triangleright d\right\rangle$ & - & $\inf _{x, y \in \Delta^{\mathcal{I}}}\left\{\left[r_{1}^{\mathcal{I}} \circ^{\otimes} \ldots \circ^{\otimes} r_{n}^{\mathcal{I}}\right](x, y) \Rightarrow r^{\mathcal{I}}(x, y)\right\} \triangleright d$ \\
Inverse role & $r^{-}$ & $\left\{(y, x) \mid(x, y) \in r^{\mathcal{I}}\right\}$ & $\left(r^{-}\right)^{\mathcal{I}}(x, y)=r^{\mathcal{I}}(y, x)$ \\
Transitive role & $\operatorname{trans}(r)$ & $r^{\mathcal{I}} \circ r^{\mathcal{I}} \subseteq r^{\mathcal{I}}$ & \\
\hline
\end{tabular}

An ontology $\mathcal{O}$ comprises of the intentional and extensional knowledge related to an application domain. The intensional knowledge, i.e. general knowledge about an application domain, is expressed via the Terminological Box (TBox) $\mathcal{T}$ and the Role Box (RBox) $\mathcal{R}$. The extensional knowledge, i.e. particular knowledge about specific situations, is expressed via an Assertional Box (ABox) $\mathcal{A}$ containing statements about individuals. Table 3 presents the syntax of statements for TBoxes and Table 4 that of ABoxes for the crisp and fuzzy variants of $\mathcal{S} \mathcal{R O} \mathcal{I} \mathcal{Q}$. As depicted in Tables 3,4, fuzzy ABoxes and TBoxes have the same syntax as their crisp counterparts, while they may also contain fuzzy assertions, fuzzy General Concept Inclusions (GCIs), and fuzzy Role Inclusions (RIs). In order to ensure decidability of the crisp DL, a set of restrictions regarding the use of roles and simple roles in GCIs and RIs is imposed, e.g. a simple role cannot subsume any transitive role, for more details see [13]. The same restrictions are also adopted for the fuzzy versions of $\mathcal{S R O \mathcal { Q }}[2,3,4]$. 
Table 4. $\mathcal{S R O} \mathcal{I} \mathcal{Q}$ ABox axioms.

\begin{tabular}{llll}
\hline & Syntax & Crisp Semantics & Fuzzy Semantics \\
\hline Concept a. & $C(a)$ & $a^{\mathcal{I}} \in C^{\mathcal{I}}$ & $C^{\mathcal{I}}\left(a^{\mathcal{I}}\right)=1$ \\
fuzzy & $C(a) \bowtie d$ & - & $C^{\mathcal{I}}\left(a^{\mathcal{I}}\right) \bowtie d$ \\
Role a. & $r(a, b)$ & $\left(a^{\mathcal{I}}, b^{\mathcal{I}}\right) \in r^{\mathcal{I}}$ & $r^{\mathcal{I}}\left(a^{\mathcal{I}}, b^{\mathcal{I}}\right)=1$ \\
$\quad$ fuzzy & $r(a, b) \bowtie d$ & - & $r^{\mathcal{I}}\left(a^{\mathcal{I}}, b^{\mathcal{I}}\right) \bowtie d$ \\
Negated role a. & $\neg r(a, b)$ & $\left(a^{\mathcal{I}}, b^{\mathcal{I}}\right) \notin r^{\mathcal{I}}$ & $r^{\mathcal{I}}\left(a^{\mathcal{I}}, b^{\mathcal{I}}\right)=0$ \\
$\quad$ fuzzy & $\neg r(a, b) \bowtie d$ & - & $\ominus r^{\mathcal{I}}\left(a^{\mathcal{I}}, b^{\mathcal{I}}\right) \bowtie d$ \\
Inequality a. & $a \neq b$ & $a^{\mathcal{I}} \neq b^{\mathcal{I}}$ & $a^{\mathcal{I}} \neq b^{\mathcal{I}}$ \\
Equality a. & $a=b$ & $a^{\mathcal{I}}=b^{\mathcal{I}}$ & $a^{\mathcal{I}}=b^{\mathcal{I}}$ \\
\hline
\end{tabular}

Example 1. Based on the concept assertion and inclusion axioms presented and explained in equation 2 we can create the following crisp ABox and TBox:

$$
\begin{aligned}
& \mathcal{A}:=\{\mathrm{CPU}(\mathrm{cpuA}), \text { Overutilized }(\mathrm{cpuA})\} \\
& \mathcal{T}:=\{\text { Server } \sqcap \exists \text { hasPart.Overutilized } \sqsubseteq \text { ServerWithLimitedResources }\}
\end{aligned}
$$

where cpuA $\in N_{I} ; \mathrm{CPU}$, Overutilized, ServerWithLimitedResources,Server $\in N_{C}$; and hasPart $\in N_{R}$. As expected Server $\sqcap \exists$ hasPart.Overutilized corresponds to a complex concept description. A fuzzy version of the previous ABox can occur if for example we add a degree of truth to the concept assertion Overutilized(cpuA). The fuzzy assertion Overutilized $(\mathrm{cpuA}) \geqslant 0.8$ states that cpuA is overutilized with a degree of at least 0.8 .

The semantics of crisp $\mathcal{S R O I} \mathcal{Q}$ are given via an interpretation $\mathcal{I}$ that is a pair $\left(\Delta^{\mathcal{I}},,^{\mathcal{I}}\right)$ consisting of a non empty set $\Delta^{\mathcal{I}}$ and an interpretation function ${ }^{\mathcal{I}}$ mapping every individual $a \in N_{I}$ onto an element $a^{\mathcal{I}} \in \Delta^{\mathcal{I}}$, every concept name $A \in N_{C}$ to a set $A^{\mathcal{I}} \subseteq \Delta^{\mathcal{I}}$, every atomic role $r \in N_{R}$ onto a relation $r^{\mathcal{I}} \subseteq \Delta^{\mathcal{I}} \times \Delta^{\mathcal{I}}$. The interpretations of complex concepts, GCIs and assertions are presented on the third column of Tables 2,3 , and 4 .

In a fuzzy extension of $\mathcal{S R O I} \mathcal{Q}$, concepts denote fuzzy sets of individuals and roles denote fuzzy binary relations. Likewise fuzzy axioms may hold to some degree. The semantics of $f-\mathcal{S R O I} \mathcal{Q}$ is given via interpretations $\mathcal{I}$ that are pairs $\left(\Delta^{\mathcal{I}},{ }^{\mathcal{I}}\right)$ consisting of a non empty set $\Delta^{\mathcal{I}}$ and an interpretation function.$^{\mathcal{I}}$ mapping every individual $a \in N_{I}$ to an element $a^{\mathcal{I}} \in \Delta^{\mathcal{I}}$, every concept name $A \in N_{C}$ onto a membership function $A^{\mathcal{I}}: \Delta^{\mathcal{I}} \rightarrow[0,1]$, every atomic role $r \in$ $N_{R}$ onto a membership function $r^{\mathcal{I}}: \Delta^{\mathcal{I}} \times \Delta^{\mathcal{I}} \rightarrow[0,1]$. In the finitely-valued setting, which we consider here, the membership function mapps to a finite subset of $[0,1]$. The interpretations of complex concepts, TBox axioms, and ABox assertions are presented on the fourth column of Tables 3,4, for the different families of fuzzy logic operators $(\otimes, \oplus, \ominus, \Rightarrow)$ presented in Table 1 . Based on the semantics reasoning services can be defined. In this paper we are interested in conjunctive query answering.

Definition 2 (Conjunctive Query for classic DLs - CQ [12]). Let $N_{V}$ be a countably infinite set of variables disjoint from $N_{C}, N_{R}$, and $N_{I}$. An atom is an expression $A(x)$ (concept atom) or $r(x, y)$ (role atom), where $A \in N_{C}$, 
$r \in N_{R}$, and $x, y \in N_{V} \cup N_{I}$. A conjunctive query $q$ is a non-empty set of atoms. Intuitively, such a set represents the conjunction of its elements. We use $\operatorname{Var}(q)$ to denote the set of variables occurring in the query $q$. Let $\mathcal{I}$ be an interpretation, $q$ a conjunctive query, and $\pi: \operatorname{Var}(q) \rightarrow \Delta^{\mathcal{I}}$ a total function, s.t. $\pi(a)=a^{\mathcal{I}}$ for all $a \in N_{I}$. We write: $\left.\mathcal{I}\right|^{\pi} C(x)$ if $\pi(x) \in C^{\mathcal{I}}$ and $\mathcal{I}={ }^{\pi} r(x, y)$ if $(\pi(x), \pi(y)) \in r^{\mathcal{I}}$. If $\mathcal{I} \models^{\pi}$ at for all atoms at $\in q$, we write $\left.\mathcal{I}\right|^{\pi} q$ and call $\pi$ a match for $\mathcal{I}$ and $q$. We say that $\mathcal{I}$ satisfies $q$ and write $\mathcal{I} \models q$ if there is a match $\pi$ for $\mathcal{I}$ and $q$. If $\mathcal{I} \models q$ for all models $\mathcal{I}$ of an ontology $\mathcal{O}$, we write $\mathcal{O} \models q$ and say that $\mathcal{O}$ entails $q$.

Finally, a union of conjunctive queries $q_{\mathrm{UCQ}}$ is a set of conjunctive queries. We write $\mathcal{O} \models q_{\mathrm{UCQ}}$ and say that $\mathcal{O}$ entails $q_{\mathrm{UCQ}}$ if for every model $\mathcal{I}$ of $\mathcal{O}$ we have that $\mathcal{I} \models q$ for some conjunctive query $q \in q_{\mathrm{UCQ}}$.

\section{Reduction to the Crisp Case}

The goal is to devise a reduction of answering UCQs over a fuzzy ontology $\mathcal{O}$ to answer UCQs over a crisp ontology $\mathcal{O}_{\mathcal{C}}$. The basic idea is that each concept and role in $\mathcal{O}$ is mapped onto a set of concepts and roles corresponding to their $\alpha$-cuts, which is the crisp set containing all elements that belong to a fuzzy set up to a given degree. For example, if the concept Overutilized in $\mathcal{O}$ maps each $\mathrm{CPU}$ to the degree to which it is overutilized, then the concept Overutilized $\geqslant 0.6$ in $\mathcal{O}_{\mathcal{C}}$ represents the set of CPUs that are overutilized to a degree of at least 0.6.

We present the reduction algorithm for the fuzzy versions of $\mathcal{S R O I} \mathcal{Q}$ corresponding to the Gödel, and Łukasiewicz based semantics. We employ the notation $[0,1]_{\mathcal{O}}$ in order to represent the finite set of degrees that appear in our ontology. We also use the notation $(a, b)_{\mathcal{O}}$ to represent the $(a, b) \cap[0,1]_{\mathcal{O}}$ subset of $[0,1]_{\mathcal{O}}$.

It has been proved for fuzzy ontologies under Gödel logics that the set of degrees of truth that must be considered for any reasoning task is the set $[0,1]_{\mathcal{O}} \cup\{0,1\}[4]$. In order to ensure that the reduction technique can be applied for $f-\mathcal{S R O I} \mathcal{Q}$ with Eukasiewicz based semantics, we need restrict to a finite number of degrees that have the form of $\left\{0, \frac{1}{n}, \ldots, \frac{n-1}{n}, 1\right\}$ where $n$ is a natural number [4]. From now on when using the notation $[0,1]_{\mathcal{O}}$ we consider that the corresponding set satisfies this restriction when referring to a Łukasiewicz based fuzzy DL.

A compact form of the reduction rules from fuzzy to crisp $\mathcal{S R O I} \mathcal{Q}$ is displayed in Table 5. It should be noted that the uppercase bold letters in this Table correspond to the conditions illustrated in Table 6 . For a detailed description of the reduction rules the reader may refer to $[3,4]$. The reduced ontology $\mathcal{O}_{\mathcal{C}}$ has the following form:

- In order to preserve the semantics of $\alpha$-cuts of atomic concepts and roles the following axioms are added to $\mathcal{T}_{\mathcal{C}}$ for every $A \in N_{C}, r \in N_{R}$ :

$$
\begin{gathered}
A_{\geqslant d_{i+1}} \sqsubseteq A_{>d_{i}} \quad A_{>d_{i}} \sqsubseteq A_{\geqslant d_{i}} \\
r_{\geqslant d_{i+1}} \sqsubseteq r_{>d_{i}} \quad r_{>d_{i}} \sqsubseteq r_{\geqslant d_{i}}
\end{gathered}
$$


Table 5. Mapping of concept and role expressions in fuzzy $\mathcal{S R O I} \mathcal{Q}$.

\begin{tabular}{|c|c|}
\hline $\begin{array}{r}\text { Reduction of concepts } \\
\text { and axioms }\end{array}$ & Gödel / Łukasiewicz \\
\hline$\rho(A, \geqslant d)$ & $A_{\geqslant d}$ \\
\hline$\rho(A, \leqslant d)$ & $\neg A_{>d}$ \\
\hline$\rho(\neg C, \geqslant d)$ & $\rho(C, \leqslant 0) / \neg \rho(C,>1-d)$ \\
\hline$\rho(\neg C, \leqslant d)$ & $\rho(C,>0) / \rho(C, \geqslant 1-d)$ \\
\hline$\rho(C \sqcap D, \geqslant d)$ & $\rho(C, \geqslant d) \sqcap \rho(D, \geqslant d) / \sqcup_{\mathbf{A}}\left(\rho\left(C, \geqslant d_{1}\right) \sqcap \rho\left(D, \geqslant d_{2}\right)\right)$ \\
\hline$\rho(C \sqcap D, \leqslant d)$ & $\rho(C, \leqslant d) \sqcup \rho(D, \leqslant d) / \rho(\neg C \sqcup \neg D, \geqslant 1-d)$ \\
\hline \multirow[t]{2}{*}{$\rho(C \sqcup D, \geqslant d)$} & $\rho(C, \geqslant d) \sqcup \rho(D, \geqslant d) /$ \\
\hline & $\rho(C, \geqslant d) \sqcup \rho(D, \geqslant d) \sqcup \sqcup_{\mathbf{B}}\left(\rho\left(C, \geqslant d_{1}\right) \sqcap \rho\left(D, \geqslant d_{2}\right)\right)$ \\
\hline$\rho(C \sqcup D, \leqslant d)$ & $\rho(C, \leqslant d) \sqcap \rho(D, \leqslant d) / \rho(\neg C \sqcap \neg D, \geqslant 1-d)$ \\
\hline$\rho(\exists r . C, \geqslant d)$ & $\exists \rho(r, \geqslant d) \cdot \rho(C, \geqslant d) / \sqcup_{\mathbf{A}}\left(\exists \rho\left(r, \geqslant d_{1}\right) \cdot \rho\left(C, \geqslant d_{2}\right)\right)$ \\
\hline$\rho(\exists r . C, \leqslant d)$ & $\forall \rho(r,>d) . \rho(C \leqslant d) / \rho(\forall r . \neg C, \geqslant 1-d)$ \\
\hline$\rho(\forall r . C, \geqslant d)$ & $\begin{array}{l}\prod_{\mathbf{C}}\left(\forall \rho\left(r, \geqslant d^{\prime}\right) \cdot \rho\left(C, \geqslant d^{\prime}\right)\right) \sqcap \Pi_{\mathbf{D}}\left(\forall \rho\left(r,>d^{\prime}\right) \cdot \rho\left(C,>d^{\prime}\right)\right) / \\
\prod_{\mathbf{E}}\left(\forall \rho\left(r, \geqslant d_{1}\right) \cdot \rho\left(C, \geqslant d_{2}\right)\right)\end{array}$ \\
\hline$\rho(\forall r . C, \leqslant d)$ & $\sqcup_{\mathbf{F}}(\exists \rho(r,>d) \cdot \rho(C, \leqslant d)) / \rho(\exists r . \neg C, \geqslant 1-d)$ \\
\hline \multirow{3}{*}{$\begin{array}{r}\rho\left(\cup_{i=1}^{m}\left\{d_{i} / o_{i}\right\}, \bowtie d\right) \\
\rho(\geq m \text { s.C }, \geqslant d)\end{array}$} & $\left\{o_{i} \mid d_{i} \bowtie d, 1 \leqslant i \leqslant m\right\}$ \\
\hline & $\geq m \rho(s, \geqslant d) \cdot \rho(C, \geqslant d) /$ \\
\hline & $\begin{aligned} \sqcup_{\mathbf{G}}\left(\exists \rho\left(s, \geqslant d_{1}\right) \cdot \rho\left(B_{1} \sqcap \rho\left(C, \geqslant e_{1}\right)\right) \sqcap\right. & \ldots \\
& \left.\sqcap \exists \rho\left(s, \geqslant d_{m}\right) \cdot\left(B_{m} \sqcap \rho\left(C, \geqslant e_{m}\right)\right)\right)\end{aligned}$ \\
\hline \multirow[t]{3}{*}{$\rho(\geq m$ s. $C, \leqslant d)$} & $\leq m-1 \rho(s,>d) \cdot \rho(C,>d) /$ \\
\hline & $\neg\left(\bigsqcup_{\mathbf{H}}\left(\exists \rho\left(s, \geqslant d_{1}\right) \cdot \rho\left(B_{1} \sqcap \rho\left(C, \geqslant e_{1}\right)\right) \sqcap \quad \ldots\right.\right.$ \\
\hline & $\left.\left.\sqcap \exists \rho\left(s, \geqslant d_{m}\right) \cdot\left(B_{m} \sqcap \rho\left(C, \geqslant e_{m}\right)\right)\right)\right)$ \\
\hline$\rho(\leq n s . C, \geqslant d)$ & $\leq n \rho(s,>0) \cdot \rho(C,>0) / \rho(\neg(\geq n+1 s . C), \geqslant d)$ \\
\hline$\rho(\leq n s . C, \leqslant d)$ & $\geq n+1 \rho(s,>0) \cdot \rho(C,>0) / \rho(\neg(\geq n+1 s . C), \leqslant d)$ \\
\hline$\rho(r, \geqslant d)$ & $r \geqslant d$ \\
\hline$\rho(r, \leqslant d)$ & $\neg r_{>d}$ \\
\hline$\rho\left(r^{-}, \geqslant d\right)$ & $r_{\geqslant d}^{-}$ \\
\hline$\rho\left(r^{-}, \leqslant d\right)$ & $\neg r_{>d}^{-}$ \\
\hline$\kappa(C(a) \bowtie d)$ & $\rho(C, \bowtie d)(a)$ \\
\hline$\kappa(r(a, b) \bowtie d)$ & $\rho(r, \bowtie d)(a, b)$ \\
\hline$\kappa(\langle C \sqsubseteq D \geqslant d\rangle)$ & $\begin{array}{l}\cup_{\mathbf{C}}\left(\rho\left(C, \geqslant d^{\prime}\right) \sqsubseteq \rho\left(D, \geqslant d^{\prime}\right)\right) \cup \bigcup_{\mathbf{D}}\left(\rho\left(C,>d^{\prime}\right) \sqsubseteq \rho\left(D,>d^{\prime}\right)\right) / \\
\bigcup_{\mathbf{I}}\left(\rho\left(C, \geqslant d_{1} \sqsubseteq \rho\left(D, \geqslant d_{2}\right)\right)\right.\end{array}$ \\
\hline$\kappa\left(\left\langle r_{1} \ldots r_{n} \sqsubseteq r \geqslant d\right\rangle\right)$ & $\begin{array}{c}\bigcup_{\mathbf{C}}\left(\rho\left(r_{1}, \geqslant d^{\prime}\right) \ldots \rho\left(r_{n}, \geqslant d^{\prime}\right) \sqsubseteq \rho\left(r, \geqslant d^{\prime}\right)\right) \cup \\
\bigcup_{\mathbf{D}}\left(\rho\left(r_{1}, \geqslant d^{\prime}\right) \ldots \rho\left(r_{n}, \geqslant d^{\prime}\right) \sqsubseteq \rho\left(r, \geqslant d^{\prime}\right)\right) / \\
\bigcup_{\mathbf{J}}\left(\rho\left(r_{1}, \geqslant d_{1}\right) \ldots \rho\left(r_{n}, \geqslant d_{n}\right) \sqsubseteq \rho\left(r, \geqslant d_{n+1}\right)\right)\end{array}$ \\
\hline
\end{tabular}

where $d_{i}, d_{i+1}$ correspond to every pair of degrees $d_{i}, d_{i+1}$ such that (i) $d_{i+1}>d_{i}$, (ii) there exists no element $e \in[0,1]_{\mathcal{O}}$ such that $d_{i+1}>e>d_{i}$, and (iii) the subscript $>1$ is not considered in any of the GCIs. For the $f_{\mathrm{E}_{n}}$

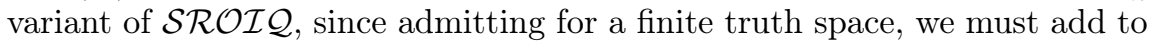
our ontology that $A_{>d_{i}} \equiv A_{\geqslant d_{i+1}}$ and $r_{>d_{i}} \equiv r_{\geqslant d_{i+1}}$.

- For each complex concept $C$ appearing in $\mathcal{O}$ the complex concept $\rho(C, \bowtie d)$ in $\mathcal{O}_{\mathcal{C}}$ represents its corresponding $\alpha$-cut. These complex concepts are inductively defined according to the set of reduction rules presented in the first part of Table 5 .

- Each ABox axiom in $\mathcal{A}$ is represented by its corresponding axiom in $\mathcal{A}_{\mathcal{C}}$ presented in the second part of Table 5 . 
Table 6. Conditions corresponding to the uppercase letters of Table 5.

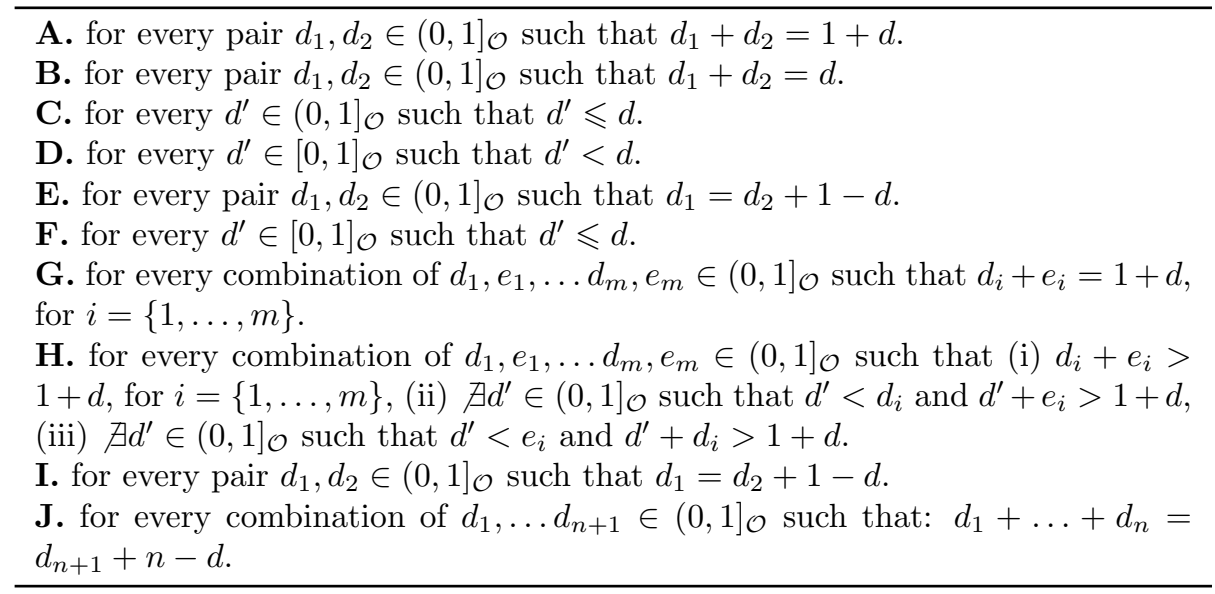

- Each TBox axiom in $\mathcal{T}$ is represented by its corresponding axiom or set of axioms in $\mathcal{T}_{\mathcal{C}}$ according to the set of reduction rules presented in the third part of Table 5 .

\section{Conjunctive Queries for Fuzzy DLs}

Our main objective is to find an algorithm for answering to conjunctive queries for fuzzy DLs based on a reduction procedure to classic ones. Different forms of conjunctive queries for fuzzy DLs have been proposed in the literature. According to [19], these are classified to queries of two different types, namely threshold conjunctive queries and general fuzzy queries. With respect to the example provided on the introduction a threshold query of the form:

$$
\text { Server }(x) \geqslant 1 \wedge \operatorname{hasPart}(x, y) \geqslant 1 \wedge \mathrm{CPU}(y) \geqslant 1 \wedge \operatorname{Overutilized}(y) \geqslant 0.6
$$

searches for all pairs of servers and CPUs such that the CPU is a part of the server and is also overutilized to a degree of at least 0.6. In contrast, a fuzzy query of the form:

$$
\operatorname{Server}(\mathrm{x}) \wedge \operatorname{hasPart}(x, y) \wedge \mathrm{CPU}(y) \wedge \operatorname{Overutilized}(y)
$$

searches for the pairs of elements that satisfy it along with the degree of satisfaction (provided that this degree is greater than 0 ).

Definition 3 (Threshold Conjunctive Query - $\mathbf{C Q}_{\theta}$ ). Let $N_{V}$ be a countably infinite set of variables disjoint from $N_{C}, N_{R}$, and $N_{I}$. A degree atom is an expression $P(\bar{X}) \triangleright d$ where $P \in N_{C} \cup N_{R}, \bar{X}$ is an ordered tuple of elements of $N_{I} \cup N_{V}$ having an arity of 1 if $P \in N_{C}$ and 2 if $P \in N_{R}, \triangleright \in\{\geqslant,>\}$, and 
$d \in(0,1]$. A Threshold Conjunctive Query has the form:

$$
\bigwedge_{i=1}^{\lambda} P_{i}\left(\bar{X}_{i}\right) \triangleright_{i} d_{i}
$$

We use VarIndivs $\left(q_{\theta}\right)$ to denote the set of variables and individuals occurring in a $C Q_{\theta}$ named $q_{\theta}$. Let $\mathcal{I}$ be an interpretation and $\pi: \operatorname{VarIndivs}(q) \rightarrow \Delta^{\mathcal{I}}$ a total function that maps each element $a \in N_{I}$ to $a^{\mathcal{I}}$. If $P_{i}^{\mathcal{I}}\left(\pi\left(\bar{X}_{i}\right)\right) \triangleright_{i} d_{i}$ for all degree atoms in $q_{\theta}$, we write $\mathcal{I} \models^{\pi} q_{\theta}$ and call $\pi$ a match for $\mathcal{I}$ and $q_{\theta}$. We say that $\mathcal{I}$ satisfies $q_{\theta}$ and write $\mathcal{I} \models q_{\theta}$ if there is a match $\pi$ for $\mathcal{I}$ and $q_{\theta}$. If $\mathcal{I} \models q_{\theta}$ for all models $\mathcal{I}$ of an ontology $\mathcal{O}$, we write $\mathcal{O} \models q_{\theta}$ and say that $\mathcal{O}$ entails $q_{\theta}$.

Definition 4 (Fuzzy Conjunctive Query - $\mathbf{C Q}_{\phi}$ ). A plain atom is an expression $P(\bar{X})$. A Fuzzy Conjunctive Query with plain atoms has the form:

$$
\bigwedge_{i=1}^{\lambda} P_{i}\left(\bar{X}_{i}\right)
$$

Let $\mathcal{I}$ be an interpretation, $q_{\phi}$ a $C Q_{\phi}, \pi$ a mapping, and $\otimes$ a fuzzy logic $t$ norm -we assume that the $t$-norms of the query and the $D L$ are the same-. If $P_{i}^{\mathcal{I}}\left(\pi\left(\bar{X}_{i}\right)\right)=d_{i}$ for all atoms in $q_{\phi}$ and $\otimes_{i=1}^{\kappa} d_{i} \geqslant d$ we write $\mathcal{I} \models^{\pi} q_{\phi} \geqslant d$ and call $\pi$ a match for $\mathcal{I}$ and $q_{\phi}$ with a degree of at least $d$. We say that $\mathcal{I}$ satisfies $q_{\phi}$ with a degree of at least $d$ and write $\mathcal{I} \models q_{\phi} \geqslant d$ if there is a corresponding match. If $\mathcal{I} \models q_{\phi} \geqslant d$ for all models $\mathcal{I}$ of a an ontology $\mathcal{O}$, we write $\mathcal{O} \models q_{\phi} \geqslant d$ and say that $\mathcal{O}$ entails $q_{\phi}$ with a degree of at least $d$. The problem of determining whether $\mathcal{O} \models q_{\phi}>d$ is defined analogously.

The query entailment problem for a $\mathrm{CQ}_{\theta}$ is to decide whether $\mathcal{O}=q_{\theta}$ for a given assignment of the variables. For $\mathrm{CQ}_{\phi} \mathrm{s}$ we may consider two variants of the query entailment problem, namely to decide whether $\mathcal{O} \models q_{\phi} \geqslant d$ for some degree $d \in(0,1]$, and to find the degree $\sup \left\{d|\mathcal{O}|=q_{\phi} \geqslant d\right\}$. Since the $f_{\mathrm{G}}, f_{\mathrm{E}_{n}}$ variants of $\mathcal{S R O I} \mathcal{Q}$ admit for the finite truth space $[0,1]_{\mathcal{O}}$ we can assume without loss of generality that the two problems can be reduced to each other. The query answering problem requests for the specific assignments that satisfy the query, thus the reduction can be achieved by testing all assignments, which give an exponential blow-up. It is well-known from crisp DLs that query entailment and query answering can be mutually reduced and that decidability and complexity results carry over [7] modulo the mentioned blow-up.

Example 5. Suppose that we have the queries described in equations 4,5, the ABox

$$
\begin{aligned}
\mathcal{A}=\left\{\operatorname{Server}\left(s_{1}\right) \geqslant 1, \operatorname{hasPart}\left(s_{1}, c p u_{1}\right) \geqslant 1,\right. \\
\left.\operatorname{CPU}\left(c p u_{1}\right) \geqslant 1, \text { Overutilized }\left(c p u_{1}\right) \geqslant 0.7\right\}
\end{aligned}
$$

and an empty TBox $\mathcal{T}$. Then the answer to equation 4 would be the pair $\left(s_{1}, c p u_{1}\right)$, while the answer to equation 5 would be $\left(s_{1}, c p u_{1}\right)$ with a degree of at least 0.7 . 
A union of $C Q_{\theta} s$ is a set of $\mathrm{CQ}_{\theta}$ s. An ontology $\mathcal{O}$ entails such a union $U_{q_{\theta}}$, i.e. $\mathcal{O} \models U_{q_{\theta}}$, when for every model $\mathcal{I} \models \mathcal{O}$ there exists some $q_{\theta} \in U_{q_{\theta}}$ such that $\mathcal{I} \equiv q_{\theta}$. Another type of union is one consisting of a set of $\mathrm{CQ}_{\phi} \mathrm{s}$. An ontology $\mathcal{O}$ entails such a union $U_{q_{\phi}}$ to a degree of at least $d \in(0,1]$, i.e. $\mathcal{O} \models U_{q_{\phi}} \geqslant d$, when for every model $\mathcal{I} \models \mathcal{O}$ there exists some $q_{\phi} \in U_{q_{\phi}}$ such that $\mathcal{I} \models q_{\phi} \geqslant d$.

Remark 6. In the context of the reduction algorithms, we focus on $\geqslant,>$ inequalities appearing in threshold/fuzzy conjunctive queries. A threshold conjunctive query with $\leqslant,<$ inequalities would be reduced to a crisp conjunctive query containing negated role atoms. Moreover, the reduction of a fuzzy conjunctive query $q_{\phi}$ with a less or equal degree $\kappa\left(q_{\phi}, \leqslant d\right)$ would be reduced to a disjunction of negated atoms. Since the problems of negated atoms and disjunctive queries have not been studied for expressive classic DLs, we focus on $\geqslant,>$ inequalities.

\section{Conjunctive Query Answering by Reduction}

In this section we provide the corresponding steps so as to solve the problem of conjunctive query answering for fuzzy DLs by taking advantage of existing crisp DL algorithms for the same problem. The solution we provide operates on the DLs and is based on the reduction techniques presented in [4,23]. We denote with $\kappa$ the reduction process from $\mathrm{CQ}_{\theta} \mathrm{s}$ and $\mathrm{CQ}_{\phi} \mathrm{s}$ queries to crisp CQs and UCQs. The reduction process operates differently for each query type.

For the $\mathrm{CQ}_{\theta}$ described in Definition 3 the reduction process takes the following form:

$$
\kappa\left(\bigwedge_{i=1}^{\lambda} P_{i}\left(\bar{X}_{i}\right) \triangleright d_{i}\right)=\bigwedge_{i=1}^{\lambda} \rho\left(P_{i}, \triangleright d_{i}\right)\left(\bar{X}_{i}\right)
$$

Since $P_{i}$ is either a concept name $A_{i} \in N_{C}$ or a role name $r_{i} \in N_{R}$ we have that $\rho\left(A_{i}, \triangleright d_{i}\right)=A_{i \triangleright d_{i}}$ or $\rho\left(r_{i}, \triangleright d_{i}\right)=r_{i \triangleright d_{i}}$ as presented on Table 5 .

The reduction process for a $\mathrm{CQ}_{\phi}$ has two inputs, the first input is the query itself and the second input is the degree that we want to examine. In addition, for $\mathrm{CQ}_{\phi}$ s the reduction process depends on the $t$-norm operator that has been adopted to provide semantics for conjunction. For the $\mathrm{CQ}_{\phi}$ described in Definition 4 the reduction process takes the form presented in equation 7 when the $\mathrm{CQ}_{\phi}$ refers to an $f_{\mathrm{G}}-\mathcal{S} \mathcal{R O} \mathcal{I} \mathcal{Q}$ ontology. When the $\mathrm{CQ}_{\phi}$ refers to an $f_{\mathrm{E}_{n}}-\mathcal{S} \mathcal{R O} \mathcal{I} \mathcal{Q}$ ontology the corresponding reduction is the union of conjunctive queries presented in equation 8 ( $\otimes$ in equation 8 stands for the Łukasiewicz t-norm operator).

$$
\begin{gathered}
\kappa\left(\bigwedge_{i=1}^{\lambda} P_{i}\left(\bar{X}_{i}\right), \geqslant d\right)=\bigwedge_{i=1}^{\lambda} \rho\left(P_{i}, \geqslant d\right)\left(\bar{X}_{i}\right) \\
\kappa\left(\bigwedge_{i=1}^{\lambda} P_{i}\left(\bar{X}_{i}\right), \geqslant d\right)=\bigcup_{\otimes_{i=1}^{\lambda} d_{i}=d \text { and } d_{i} \in[0,1]_{\mathcal{O}}}\left\{\bigwedge_{i=1}^{\lambda} \rho\left(P_{i}, \geqslant d_{i}\right)\left(\bar{X}_{i}\right)\right\}
\end{gathered}
$$


Example \%. The reduced form of the $\mathrm{CQ}_{\theta}$ presented in equation 4 follows in equation 9. The reduced form of the $\mathrm{CQ}_{\phi}$ in equation 5 for the degree of at least 0.75 for the $f_{\mathrm{G}}-\mathcal{S} \mathcal{R O} \mathcal{I} \mathcal{Q}$ logic follows in equation 10 . Finally if we consider the $f_{\mathrm{E}_{4}}-\mathcal{S} \mathcal{R O} \mathcal{I} \mathcal{Q}$ logic we have that $[0,1]_{\mathcal{O}}=\{0,0.25,0.5,0.75,1\}$ and the reduced form of equation 5 for the degree of at least 0.75 is the UCQ presented in equation 11:

$$
\begin{aligned}
& \text { Server } \geqslant 1(x) \wedge \text { hasPart } \geqslant 1_{\geqslant}(x, y) \wedge \mathrm{CPU}_{\geqslant 1}(y) \wedge \text { Overutilized }_{\geqslant 0.6}(y) \\
& \text { Server } \geqslant 0.75(x) \wedge \text { hasPart } \geqslant 0.75(x, y) \wedge \mathrm{CPU}_{\geqslant 0.75}(y) \wedge \text { Overutilized } \geqslant 0.75(y) \\
& \left\{\text { Server } \geqslant 0.75(x) \wedge \text { hasPart } \geqslant 1(x, y) \wedge \mathrm{CPU}_{\geqslant 1}(y) \wedge \text { Overutilized } \geqslant 1(y)\right\} \cup \\
& \left\{\text { Server } \geqslant 1(x) \wedge \text { hasPart } \geqslant 0.75(x, y) \wedge \mathrm{CPU}_{\geqslant 1}(y) \wedge \text { Overutilized } \geqslant 1(y)\right\} \cup \\
& \left\{\text { Server }_{21}(x) \wedge \text { hasPart }_{21}(x, y) \wedge \mathrm{CPU}_{\geqslant 0.75}(y) \wedge \text { Overutilized }_{\geqslant 1}(y)\right\} \cup \\
& \left\{\text { Server } \geqslant 1(x) \wedge \text { hasPart } \geqslant 1(x, y) \wedge \mathrm{CPU}_{\geqslant 1}(y) \wedge \text { Overutilized } \geqslant 0.75(y)\right\}
\end{aligned}
$$

The following Theorem states that our query reduction algorithm is sound and complete. Since we consider the $f_{\mathrm{G}}, f_{\mathrm{E}_{n}}$ variants of $\mathcal{S R O I} \mathcal{I}$ the theorem applies for these DLs and only. A generalization of the theorem follows in Corollaries 9,10 .

Theorem 8. Let $\mathcal{O}_{\mathcal{C}}$ be the crisp version of the fuzzy Ontology $\mathcal{O}$ such that $\kappa(\mathcal{O})=\mathcal{O}_{\mathcal{C}}, q_{\theta}$ be a $C Q_{\theta}$ and $\kappa\left(q_{\theta}\right)$ its form obtained by the reduction, $q_{\phi}$ is a $C Q_{\phi}$ and $\kappa\left(q_{\phi}, \geqslant d\right)$ its reduced form for the degree $d \in[0,1]_{\mathcal{O}}$. Then the following equivalences apply:

1. $\mathcal{O} \models q_{\theta} \Leftrightarrow \mathcal{O}_{\mathcal{C}} \models \kappa\left(q_{\theta}\right)$

2. $\mathcal{O} \models q_{\phi} \geqslant d \Leftrightarrow \mathcal{O}_{\mathcal{C}} \models \kappa\left(q_{\phi}, \geqslant d\right)$.

Proof (Sketch). In order to prove that $\mathcal{O}_{\mathcal{C}} \models \kappa\left(q_{\theta}\right) \Rightarrow \mathcal{O} \models q_{\theta}$, we build for every model $\mathcal{I}$ of $\mathcal{O}$ a non fuzzy interpretation $\mathcal{I}_{\mathcal{C}}=\left\{\Delta^{\mathcal{I}_{\mathcal{C}}},{ }^{\mathcal{I}_{\mathcal{C}}}\right\}$ as follows:

$$
\begin{aligned}
\Delta^{\mathcal{I}_{\mathcal{C}}} & =\Delta^{\mathcal{I}} & A_{\triangleright d}^{\mathcal{I}_{\mathcal{C}}} & =\left\{\beta \mid A^{\mathcal{I}}(\beta) \triangleright d\right\} \\
a^{\mathcal{I}_{\mathcal{C}}} & =a^{\mathcal{I}} & r_{\triangleright d}^{\mathcal{I}_{\mathcal{C}}} & =\left\{(\beta, \gamma) \mid r^{\mathcal{I}}(\beta, \gamma) \triangleright d\right\} .
\end{aligned}
$$

It is shown in [3,4] that $\mathcal{I}_{\mathcal{C}}$ is a model of the crisp ontology $\mathcal{O}_{\mathcal{C}}$. Since $\mathcal{I}_{\mathcal{C}} \models \mathcal{O}_{\mathcal{C}}$ and $\mathcal{O}_{\mathcal{C}} \models \kappa\left(q_{\theta}\right)$ it applies that $\mathcal{I}_{\mathcal{C}} \models \kappa\left(q_{\theta}\right)$. Based on the construction of $\mathcal{I}_{\mathcal{C}}$ and the form of $\kappa\left(q_{\theta}\right)$ (equation 6 ), it can be verified that $\mathcal{I} \models q_{\theta}$ must also apply. It can be shown in a similar way that $\mathcal{O} \models q_{\phi} \geqslant d \Rightarrow \mathcal{O}_{\mathcal{C}} \models \kappa\left(q_{\phi}, \geqslant d\right)$.

The proof of the opposite direction is performed by building a fuzzy interpretation $\mathcal{I}$ for each model $\mathcal{I}_{\mathcal{C}}$ of $\mathcal{O}_{\mathcal{C}}$ as follows:

$$
\begin{aligned}
& \Delta^{\mathcal{I}}=\Delta^{\mathcal{I}_{\mathcal{C}}} \quad A^{\mathcal{I}}(\beta)=\sup \left\{d \mid \beta \in A_{\geqslant d}^{\mathcal{I}_{\mathcal{C}}}\right\} \cup\left\{d_{+} \mid \beta \in A_{>d}^{\mathcal{I}_{\mathcal{C}}}\right\} \\
& a^{\mathcal{I}}=a^{\mathcal{I}_{\mathcal{C}}} \quad r^{\mathcal{I}}(\beta, \gamma)=\sup \left\{d \mid(\beta, \gamma) \in r_{\geqslant d}^{\mathcal{I}_{\mathcal{C}}}\right\} \cup\left\{d_{+} \mid(\beta, \gamma) \in r_{>d}^{\mathcal{I}_{\mathcal{C}}}\right\}
\end{aligned}
$$

where the degree $d_{+}$for the language of $f_{\mathrm{G}}-\mathcal{S R O} \mathcal{I} \mathcal{Q}$ is defined to be some degree in $[0,1]$ such that $d<d_{+}$and there exists no $d^{\prime} \in[0,1]_{\mathcal{O}}$ with $d<d^{\prime}<d_{+}$. For the language of $f_{\mathrm{E}_{n}}-\mathcal{S R O \mathcal { I } Q}$ the degree $d_{+}$is defined in a similar way with the main difference that it has to belong to $[0,1]_{\mathcal{O}}$. 
Corollary 9. If (i) there is a reduction technique from a fuzzy $D L f$ - $\mathcal{L}$ to a crisp $D L \mathcal{L}$, (ii) for each model $\mathcal{I}$ of an ontology $\mathcal{O}$ in the $D L$ of $f$ - $\mathcal{L}$ there exists a corresponding model $\mathcal{I}_{\mathcal{C}}$ for the reduced ontology $\mathcal{O}_{\mathcal{C}}$ that can be built based on equation 12, (iii) for each model $\mathcal{I}_{\mathcal{C}}$ of the reduced ontology $\mathcal{O}_{\mathcal{C}}$ there exists a corresponding model $\mathcal{I}$ for the initial ontology $\mathcal{O}$ that can be built based on equation 13, (iv) there exists a query answering algorithm for the DL of $f-\mathcal{L}$ then: the reduction technique can be applied in order to answer to threshold queries for the $D L$ of $f$ - $\mathcal{L}$.

Corollary 10 (Generalization of Corollary 9). If $f$ - $\mathcal{L}$ and $\mathcal{L}$ satisfy criteria (i), (ii), (iii) presented in Corollary 9 and (iv) $f$ - $\mathcal{L}_{\text {sub }}$ is a sub-language of $f-\mathcal{L}$ (v) $f$ - $\mathcal{L}_{\text {sub }}$ can be reduced to a sub-language $\mathcal{L}_{\text {sub }}$ of $\mathcal{L}$ for which there exists a query answering algorithm, then the reduction technique can be applied in order to answer to threshold queries for the language of $f$ - $\mathcal{L}_{\text {sub }}$.

Since there are algorithms for conjunctive query answering for the DLs $\mathcal{S H I Q}$

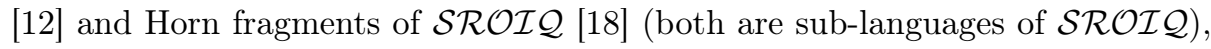
we can apply the reduction technique for conjunctive query answering for the language of $f-\mathcal{S H I} \mathcal{Q}$ and Horn fragments of $f-\mathcal{S R O I} \mathcal{Q}$ (where $f$ correspond to one of $f_{\mathrm{G}}, f_{\mathrm{E}}$ fuzzy logics).

\section{Complexity Results}

Complexity in the size of $\mathcal{O}$. According to [3], the reduction process for the DL $f_{\mathrm{G}}-\mathcal{S} \mathcal{R O \mathcal { I } Q}$ creates an ontology $\mathcal{O}_{\mathcal{C}}$ that has size $O\left(|\mathcal{O}|^{2}\right)$ compared to the initial ontology $\mathcal{O}$. If we combine the latter with the facts that: i) "conjunctive query entailment in the crisp $\mathcal{S H I} \mathcal{Q}$ can be decided in time exponential in the size of the ontology [12]" $(\dagger)$ ii) the language $\mathcal{S H I \mathcal { Q }}$ is a sublanguage of $\mathcal{S R O \mathcal { L }}$, we get an exponential complexity with respect to the size of the initial ontology. Regarding the $f_{E_{n}}-\mathcal{S R O I} \mathcal{Q} \mathrm{DL}$, the size of the resulting ontology $\mathcal{O}_{\mathcal{C}}$ is $O\left(|\mathcal{O}|\left|[0,1]_{\mathcal{O}}\right|^{k}\right)$ in case no number restrictions occur in $\mathcal{O}$, where $k$ is the maximal depth of the concepts appearing in $\mathcal{O}$ (proof in [4]). Intuitively the depth of some $A \in N_{C}$ is 1 while the depth of $\exists r$. $(\forall r . A)$ is 3 . The latter results are discouraging, with the absence of number restrictions the size of $\mathcal{O}_{\mathcal{C}}$ may become exponential w.r.t. the size of $\mathcal{O}$. If we combine these results with fact $(\dagger)$ we get a double exponential upper bound for threshold query answering w.r.t. a $f_{\mathrm{E}_{n}}-\mathcal{S H I} \mathcal{I}$ ontology, even with the absence of number restrictions.

Complexity in the size of the query. We examine the complexity w.r.t. the size of the examined threshold/fuzzy conjunctive query. Suppose that $\kappa\left(q_{\theta}\right)$ is the reduced form of a threshold conjunctive query denoted with $q_{\theta}$. We have that the size of $\kappa\left(q_{\theta}\right)$ is linear to the size of $q_{\theta}$. The size of the reduced form $\kappa\left(q_{\phi}, \geqslant d\right)$ of a fuzzy conjunctive query also remains linear w.r.t. the size of the initial fuzzy query $q_{\phi}$ if we consider the $f_{\mathrm{G}}-\mathcal{S H \mathcal { I } Q}$ semantics. For fuzzy conjunctive queries under the Eukasiewicz semantics, the size of $\kappa\left(q_{\phi}, \geqslant d\right)$ belongs to the complexity class $O\left(\left|[0,1]_{\mathcal{O}}\right|^{k-1}\right)$ where $k$ is the number of conjuncts in $q_{\phi}$. Therefore it is 
exponential compared to the size of $q_{\phi}$. If we combine the latter results with

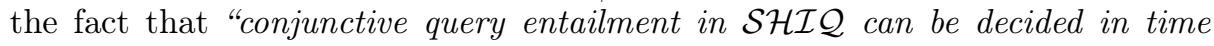
double exponential in the size of the query [12]" we get a double exponential complexity for threshold query answering and fuzzy conjunctive query answering w.r.t. $f_{\mathrm{G}}-\mathcal{S H \mathcal { L }}$ ontologies. Otherwise, we get a triple exponential upper bound

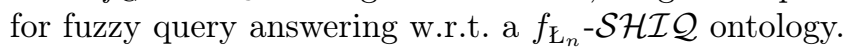

\section{Generalizing the Query Component}

So far we have examined the reduction technique for answering threshold and fuzzy CQs. These two types of queries are immediate extensions of the classic CQ problem. Nevertheless, the existence of degrees may lead to more general forms of fuzzy CQs in which the score of a query is computed via a monotone scoring function:

Example 11. Lets extend the query in Equation 5 by asking for servers that have overutilized CPU and RAM memory, while the utilization of the CPU is more important than that of the RAM memory. The resulting query will take the form:

$$
\begin{aligned}
\operatorname{Server}(\mathrm{x}) & \wedge \operatorname{hasPart}(x, y) \wedge \mathrm{CPU}(y) \wedge \operatorname{hasPart}(x, z) \wedge \\
\operatorname{RAM}(z) & \wedge \frac{0.6 \cdot \operatorname{Overutilized}(y)+0.4 \cdot \operatorname{Overutilized}(z)}{2}
\end{aligned}
$$

where the fraction corresponds to an aggregation scoring function that takes into account the degree of overutilization of a CPU and the degree of overutilization of a RAM memory with weights 0.6 and 0.4 respectively.

Such kind of queries have already been defined in the literature $[19,26,27]$ and the question is if the reduction technique can be applied to answer them. By taking account the fact that the reduction technique works on finite valued fuzzy DLs, these problems can be solved by considering for all possible combinations of degrees in $[0,1]_{\mathcal{O}}$. We consider the previous example for the $f_{\mathrm{Ł}_{n}}-\mathcal{S R O I} \mathcal{Q}$ with $[0,1]_{\mathcal{O}}=\{0,0.25,0.5,0.75,1\}$, where the concepts Server, CPU, RAM and the role hasPart are essentially crisp. The (crisp) conjunctive query

$$
\begin{aligned}
\text { Server }_{\geqslant 1}(x) \wedge \operatorname{hasPart}_{\geqslant 1}(x, y) \wedge \mathrm{CPU}_{\geqslant 1}(y) \wedge \text { hasPart }_{\geqslant 1}(x, z) \wedge \\
\operatorname{RAM}_{\geqslant 1}(z) \wedge \text { Overutilized }_{\geqslant 0.25}(y) \wedge \text { Overutilized }_{\geqslant 0.75}(z)
\end{aligned}
$$

if applied on the reduced ontology will return the triples of Server, CPU, and RAM that satisfy the query in Equation 14 with a degree greater or equal than 0.45 (i.e. $0.6 \cdot 0.25+0.4 \cdot 0.75$ ).

Another interesting problem, specific to fuzzy DLs, is the top- $k$ query answering problem presented in $[25,26,27]$. This variation of the fuzzy query answering problem focuses on the $k$ answers with the highest degrees of satisfaction. In a naive approach to solve this problem, the reduction technique for $\mathrm{CQ}_{\phi} \mathrm{s}$ can be iteratively applied starting from the highest to the lowest degrees in $[0,1]_{\mathcal{O}}$ until the limit of $k$ answers is reached. It has to be investigated if a more sophisticated approach can be adopted to solve this problem. 


\section{Related Work}

Non-fuzzy representations of fuzzy DLs have been extensively studied for several families of DLs that can be classified based on their fuzzy and DL parts. Reduction techniques have been proposed in $[23,22,2]$ for the DLs of $f-\mathcal{A L C H}$, $f-\mathcal{S H O I N}$, and $f-\mathcal{S} \mathcal{R O} \mathcal{I} \mathcal{Q}$, that are based on the Zadeh fuzzy logic semantics. An experimental evaluation of the reduction technique for the DL of $f_{K D^{-}} \mathcal{S H \mathcal { I } N}$ is presented in [11]. A reduction procedure for the $\mathcal{S R O I} \mathcal{Q}$ DL under Gödel semantics is considered in [3], while in [4] the reduction technique for the finitely many valued Łukasiewicz fuzzy Description Logic $f_{\mathrm{E}_{n}}-\mathcal{S R O \mathcal { O } Q}$ is studied. Based on a different approach, a family of fuzzy DLs using $\alpha$-cuts as atomic concepts and roles is considered in [15], while a generalization of existing approaches where a finite totally ordered set of linguistic terms or labels is assumed is presented in $[5]$.

Conjunctive query answering for fuzzy DLs has been mostly studied for the fuzzy DL-Lite family of DLs. In $[24,25]$ the problem of evaluating ranked top-k queries in the Description logic fuzzy DL-Lite is considered, while a variety of query languages by which a fuzzy DL-Lite knowledge base can be queried is presented in [19]. Tableaux based approaches for conjunctive query answering have also been studied. A tableaux algorithm for conjunctive query answering for the language of fuzzy CARIN, a knowledge representation language combining the DL $f_{\mathrm{Z}}-\mathcal{A} \mathcal{L C N \mathcal { R }}$ with Horn rules, is provided in [17] . An algorithm for answering expressive fuzzy conjunctive queries is presented in $[10,9]$. The algorithm allows the occurrence of both lower bound and the upper bound of thresholds in a query atom, over the DLs $f_{\mathrm{Z}}-\mathcal{A L C N}$, and $f_{\mathrm{Z}}-\mathcal{S H \mathcal { I N }}$. Finally, practical approach for storing and querying fuzzy knowledge in the semantic web have been investigated in [21].

\section{Conclusions and Future Work}

This paper describes how non fuzzy representation of fuzzy DLs can be adopted in order to solve the threshold and fuzzy conjunctive query answering problems. Specifically, the previously mentioned problems on fuzzy DLs are reduced to their equisatisfiable conjunctive query (or union of conjunctive queries) answering problems on crisp DLs. The correctness of the suggested techniques is proved and their complexity is studied for different fuzzy variants of the $\mathcal{S R O I} \mathcal{Q}$ DL. As far as we know no similar theoretical results have been presented. The proofs rely on the fact that each model of a fuzzy ontology $\mathcal{O}$ can be mapped to a model of its reduced crisp form $\mathcal{O}_{\mathcal{C}}$ and vice versa (soundness and completeness of the reduction technique), while they are based on the structure of the two constructed models. To verify the correctness of our approach we have extended the correctness proofs sketched in [4]. Therefore this paper can be considered complementary to the existing literature on non fuzzy representation of fuzzy DLs.

Our current line of works involves implementing the reduction techniques for

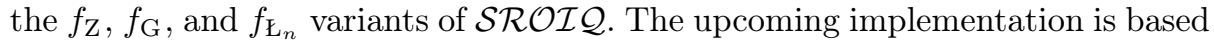


on the HermiT OWL Reasoner [20] extended with the OWL BGP SPARQL wrapper [14] that is used for conjunctive query answering. Future work involves evaluating the proposed reduction techniques on real data, studying their performance, and examining if available optimizations techniques for fuzzy and crisp DLs can be applied to improve the performance of these algorithms. Another interesting line of work involves applying these reduction based threshold and fuzzy query answering algorithms for the more general family of finite lattice based fuzzy DLs presented in [6].

\section{References}

1. Baader, F., Borgwardt, S., Peñaloza, R.: On the decidability status of fuzzy $\mathcal{A L C}$ with general concept inclusions. Journal of Philosophical Logic (2014)

2. Bobillo, F., Delgado, M., Gómez-Romero, J.: Optimizing the crisp representation of the fuzzy Description Logic $\mathcal{S} \mathcal{R} \mathcal{O} \mathcal{I}$. In: Uncertainty Reasoning for the Semantic Web I, pp. 189-206. Springer (2008)

3. Bobillo, F., Delgado, M., Gómez-Romero, J., Straccia, U.: Fuzzy Description Logics under Gödel semantics. International Journal of Approximate Reasoning 50(3), 494-514 (2009)

4. Bobillo, F., Straccia, U.: Reasoning with the finitely many-valued Łukasiewicz fuzzy Description Logic $\mathcal{S} \mathcal{R O} \mathcal{I} \mathcal{Q}$. Information Sciences 181(4), 758-778 (2011)

5. Bobillo, F., Straccia, U.: Finite fuzzy Description Logics and crisp representations. In: Uncertainty Reasoning for the Semantic Web II, pp. 99-118. Springer (2013)

6. Borgwardt, S., Peñaloza, R.: Consistency reasoning in lattice-based fuzzy Description Logics. International Journal of Approximate Reasoning (2013)

7. Calvanese, D., De Giacomo, G., Lenzerini, M.: On the decidability of query containment under constraints. In: Proceedings of the seventeenth ACM SIGACTSIGMOD-SIGART symposium on Principles of database systems. pp. 149-158. ACM (1998)

8. Cerami, M., Straccia, U.: On the (un)decidability of fuzzy Description Logics under Łukasiewicz $t$-norm. Information Sciences 227, 1-21 (2013)

9. Cheng, J., Ma, Z., Zhang, F., Wang, X.: Deciding query entailment for fuzzy $\mathcal{S H \mathcal { H } N}$ ontologies. In: Proceedings of the 4th Asian Conference on The Semantic Web, pp. 120-134. ASWC '09, Springer (2009)

10. Cheng, J., Ma, Z., Zhang, F., Wang, X.: Deciding query entailment in fuzzy Description Logic knowledge bases. In: Database and Expert Systems Applications. pp. 830-837. Springer (2009)

11. Cimiano, P., Haase, P., Ji, Q., Mailis, T., Stamou, G., Stoilos, G., Tran, D.T., Tzouvaras, V.: Reasoning with large A-Boxes in fuzzy Description Logics using DL reasoners: an experimental evaluation. In: Proceedings of the ESWC Workshop on Advancing Reasoning on the Web: Scalability and Commonsense (2008)

12. Glimm, B., Horrocks, I., Lutz, C., Sattler, U.: Conjunctive query answering for the Description Logic $\mathcal{S H \mathcal { I }}$. Journal of Artificial Intelligence Research 31, 157-204 (2008)

13. Horrocks, I., Kutz, O., Sattler, U.: The even more irresistible $\mathcal{S} \mathcal{R O} \mathcal{I} \mathcal{Q}$. In: Proc. of the 10th International Conference of Knowledge Representation and Reasoning (KR-2006). vol. 6, pp. 57-67 (2006)

14. Kollia, I., Glimm, B.: Optimizing SPARQL query answering over OWL ontologies. Journal of Artificial Intelligence Research 48, 253-303 (2013) 
15. Li, Y., Xu, B., Lu, J., Kang, D., Wang, P.: A family of extended fuzzy Description Logics. In: Computer Software and Applications Conference. vol. 1, pp. 221-226. IEEE (2005)

16. Mailis, T., Peñaloza, R., Turhan, A.Y.: Conjunctive query answering in finitelyvalued fuzzy Description Logics. Tech. rep., Chair of Automata Theory, Institute of Theoretical Computer Science, Technische Universität Dresden, Dresden, Germany (2014)

17. Mailis, T., Stoilos, G., Stamou, G.: Expressive reasoning with Horn rules and fuzzy Description Logics. Knowledge and Information Systems 25(1), 105-136 (2010)

18. Ortiz, M., Rudolph, S., Šimkus, M.: Query answering in the Horn fragments of the description logics $\mathcal{S H O I Q}$ and $\mathcal{S} \mathcal{R O} \mathcal{I} \mathcal{Q}$. In: Proceedings of the Twenty-Second international joint conference on Artificial Intelligence. vol. 2, pp. 1039-1044. AAAI Press (2011)

19. Pan, J.Z., Stamou, G.B., Stoilos, G., Thomas, E.: Expressive querying over fuzzy DL-Lite ontologies. In: 20th International Workshop on Description Logics (2007)

20. Shearer, R., Motik, B., Horrocks, I.: Hermit: A highly-efficient OWL reasoner. In: Proc. of the 5th Int. Workshop on OWL: Experiences and Directions (OWLED). Karlsruhe, Germany (2008)

21. Simou, N., Stoilos, G., Tzouvaras, V., Stamou, G.B., Kollias, S.D.: Storing and querying fuzzy knowledge in the Semantic Web. In: 7th International Workshop on Uncertainty Reasoning For the Semantic Web. Karlsruhe, Germany (2008)

22. Stoilos, G., Stamou, G.B.: Extending fuzzy Description Logics for the Semantic Web. In: 3rd International Workshop of OWL: Experiences and Directions. Innsbruck (2007)

23. Straccia, U.: Transforming fuzzy Description Logics into classical Description Logics. In: Logics in Artificial Intelligence, pp. 385-399. Springer (2004)

24. Straccia, U.: Answering vague queries in fuzzy DL-Lite. In: Proceedings of the 11th International Conference on Information Processing and Management of Uncertainty in Knowledge-Based Systems,(IPMU-06). pp. 2238-2245 (2006)

25. Straccia, U.: Towards top- $k$ query answering in Description Logics: the case of DLLite. In: Logics in Artificial Intelligence, pp. 439-451. Lecture Notes in Computer Science, Springer (2006)

26. Straccia, U.: Foundations of Fuzzy Logic and Semantic Web Languages. CRC Press (2013)

27. Straccia, U.: On the top-k retrieval problem for ontology-based access to databases. In: Flexible Approaches in Data, Information and Knowledge Management, pp. 95-114. Springer (2014) 